\title{
RECENSIÓN
}

\section{GESTIÓN DIARIA DEL HOSPITAL}

\author{
M.A. Asenjo, L.L. Bohigas*, A. Trilla*, A. Prat, M.T. Arbués, A.M. Ballesta, M. Bruguera, J. \\ Grau, F. Lamata, N.H. Larroca, A. Lobo, J. Millá, J. Rodés y J. Sánchez-Caro. \\ Año 1998. \\ Masson. Barcelona. \\ ISBN 84-458-0679-3. \\ 615 páginas.
}

Recensión: Alfredo Rivas Antón. Plan Nacional del Sida. Dirección General de Salud Pública. Ministerio de Sanidad y Consumo

El libro que comentamos impresiona, desde el principio, por el elenco de autores, todos ellos conocidos publicistas sobre todo en el área de la administración sanitaria y algunos más en concreto en el área de la gestión hospitalaria.

En el prefacio queda patente la intención holística de la publicación, que pudiera no cuadrar con el titulo, «Gestión diaria del hospital», pues, en principio, podría pensarse que el hospital estaba ya proyectado, planificado, construido y dotado de medios personales y equipamiento antes de comenzar el día a día y, en consecuencia, se trataría de gestionar este hospital, ya en desarrollo, ya en marcha, según las mejores técnicas de gestión disponibles. No obstante, seguramente con la intención de enmarcar el tema, los distintos capítulos abordan los aspectos previos.

El «Derecho y salud. Responsabilidades profesionales» es una magnifica exposición del estado de la materia, cada vez más compleja y que afecta al ejercicio de las profesiones sanitarias. Con el estilo llano y brillante que lo caracteriza, Sánchez-Caro pone en antecedentes suficientes a «los no licenciados en derecho». «Sistemas hospita- larios eficientes») permite al autor, Asenjo, desarrollar algunas de las premisas del enunciado. El capítulo 3. "Gestión estratégica», escrito por J. Mauri i Santuré, define y desarrolla lo que debe ser un plan estratégico en el hospital bien gestionado. «Marketing hospitalario"), redactado por F. Lamata, determina qué podemos entender por mercadotecnia, en un contexto general y en el contexto concreto del hospital.

«Reingeniería: el hospital orientado al paciente), a cargo de J. Grau Sociats y A. Trilla García, comienza dando por hecho que habrá competencia entre hospitales y trata de introducir estos en la dinámica de cambios radicales. «Gestión Clínica: Una visión actual del hospital», tiene por autora a M. Casas que lo desarrolla enfatizando la importancia de la información. El capítulo «Gestión de los recursos humanos») elaborado por M.T. Arbués Visús y Ll. Tarín Martínez parte de la posibilidad de seleccionar al personal, incluyendo como apéndice un «Calculo de la plantilla de personal». «Organización médica», a cargo de M. Bruguera hace breve historia del tema y se extiende sobre la organización de los médicos dentro del hospital y la elección de sus representantes. «Organización y análisis del trabajo de enfermería» escrito por $\mathrm{C}$. Yarritu Fez. y L. Bohigas S. hace un recorrido 
por las funciones de la enfermería y se adentra en cálculos de costes. El capítulo «Servicios centrales: aspectos generales», viene firmado por A. Ordinzas Bauza, J. Sau Giralt y Asenjo en el se hace una introducción de lo que se desarrollará en los seis capítulos siguientes. El capítulo Laboratorios: diagnostico biológico» producto de la colaboración entre A.M. Ballesta Gimeno, J.L. Bedini Chiesa y M.J. Insa Hernández es una exhaustiva descripción de las pautas que ha de seguir un laboratorio.

El capítulo «Diagnóstico por la imagen», de F. Pons Pons y J. Setoain Quinquer hace un breve repaso de técnicas, organización y uso racional. «Servicio de Psiquiatría: unidades de medicina psicosomática y/o psiquiatría de enlace», escrita por A. Lobo Sauté define la psiquiatría de enlace y esboza la organización idónea. El capítulo «Servicio de urgencias» desarrollado por J. Millá Santos describe los cambios últimos de estos servicios y las actuales necesidades. «Gestión del servicio de farmacia» redactado por J. Ribas Sala y C. Codina Jané, además de programar el servicio, lleva como apéndice un apartado sobre gestión económica.

El capítulo $16 .^{\circ}$ esta dedicado a «Logística hospitalaria» por J. Cussó Palacín, en el se trata el tema con especial énfasis en la parte informática.

El capítulo $17 .^{\circ}$ «Gestión de la investigación biomédica en los hospitales» está redactado por A. Trilla García poniendo como ejemplo la Fundació Cliníc per a la Recerca Biomédica.

El capítulo $18 .^{\circ}$ «Calidad asistencial», de R. Lledó Rodríguez y A. Prat Marín», define calidad asistencial y proyecta un programa de evaluación y gestión de esta.

El capítulo $19 .^{\circ}$ «Acreditación hospitalaria», escrito por L1. Bohigas, tras una introducción histórica determina un programa de acreditación adecuado.

El capítulo $200^{\circ}$ «Evaluación de tecnologías sanitarias», corre a cargo del responsa- ble de la unidad en el Instituto de Salud Carlos III, J.L. Conde Olasagasti que resalta los aspectos objeto de la evaluación tecnológica.

El capítulo $21 .^{\circ}$ «Información clínica en la gestión del hospital», escrito por A. Conesa González, explicita la importancia de la documentación, la necesidad de su recogida rigurosa y de su tratamiento ágil y confidencial.

El capítulo 22. ${ }^{\circ}$ «Epidemiología hospitalaria y evaluación de servicios sanitarios», con A. Trilla García y Asenjo de autores, hace un amplio recorrido por las epidemiologías de aplicación en el hospital, entroncando con la evaluación de servicios y medición de resultados asistenciales.

El capítulo 23. ${ }^{\circ}$ de M.A. Broggi Trias «Bioética como ayuda a la clínica», incide en los problemas éticos surgidos o agravados con los avances tecnológicos aplicados a la medicina.

El capítulo 24. "Contabilidad, control de gestión y cuadro de mando», que redactan Ll. Bohigas y Asenjo, desarrolla la contabilidad y los cuadros de mando imprescindibles.

El capítulo $25 .^{\circ}$ «Arquitectura hospitalaria. Remodelaciones», elaborado por E. Guilera, parte de la base de justificarse, solo, una remodelación para aumentar la eficiencia.

En el capítulo $26^{\circ}$ el libro hace un paréntesis en el día a día hospitalario para dar cabida a un articulo de N.H. Larroca sobre la asistencia sanitaria en Argentina, bajo el epígrafe «El modelo sanitario iberoamericano: el modelo argentino».

Termina la exposición temática del libro con una prospectiva a cargo del editor M.A. Asenjo, en este último capítulo (el 27. ${ }^{\circ}$ ) Asenjo hace consideraciones sobre lo que pueden ser los futuros, inmediato y mediato de los hospitales. Pero aún le restan al libro que recensamos otras 150 páginas interesantes de apéndices, que pueden ser de utilidad 
para aplicar en la planificación o en la gestión más del momento a momento. Apéndice que recuerda en un amplio cuadro de José Ramón Rodríguez, las reformas de la gestión hospitalaria de la década 85-96 en los países con Sistema Nacional de Salud, en el que se muestra, a las claras, las dificultades de la tarea objeto de esta publicación.

Concretando en dos frases: consideramos de interés el libro y creemos será referencia para la gestión hospitalaria. 